Aleksander Maliszewski, Department of Mathematics, Pedagogical University, pl. Weyssenhoffa 11, 85-072 Bydgoszcz, Poland. e-mail:

AMal@wsp. bydgoszcz.pl

\title{
ON PRODUCTS OF BOUNDED QUASI-CONTINUOUS FUNCTIONS
}

\begin{abstract}
The goal of this paper is to characterize the functions that can be written as the product of bounded quasi-continuous functions.
\end{abstract}

\section{Preliminaries}

The letters $\mathbb{R}, \mathbb{Z}$, and $\mathbb{N}$ denote the real line, the set of integers and the set of positive integers, respectively. For each $A \subset \mathbb{R}$ we use the symbols $\operatorname{int} A$, cl $A, \operatorname{bd} A, \lambda(A)$, and $\chi_{A}$ to denote the interior, the closure, the boundary, the outer Lebesgue measure and the characteristic function of $A$, respectively. We say that a set $A \subset \mathbb{R}$ is simply open [1] if $A \backslash \operatorname{int} A$ is nowhere dense. The symbol $\varrho$ denotes the (Euclidean) distance in $\mathbb{R}$.

The word function denotes a mapping from $\mathbb{R}$ into $\mathbb{R}$ unless otherwise explicitly stated. Let $f$ be a function. For each $y \in \mathbb{R}$ the symbol $[f<y]$ stands for $\{x \in \mathbb{R}: f(x)<y\}$; similarly we define the symbols $[f \leq y]$, [ $f>y$ ], etc. If a set $A \subset \mathbb{R}$ is nonempty, then let $\omega(f, A)$ be the oscillation of $f$ on $A$; i.e., $\omega(f, A)=\sup \{|f(x)-f(t)|: x, t \in A\}$. For each $x \in \mathbb{R}$ we write $\omega(f, x)=\lim _{\delta \rightarrow 0^{+}} \omega(f,(x-\delta, x+\delta))$; i.e., $\omega(f, x)$ is the oscillation of $f$ at $x$. The symbols $\mathcal{C}_{f}$ and $\mathcal{D}_{f}$ denote the set of points of continuity of $f$ and the set of points of discontinuity of $f$, respectively. For each $C \subset \mathbb{R}$ and each $x \in \operatorname{cl}\left(\mathcal{C}_{f} \cap C\right)$ we put $\underline{\operatorname{LIM}}(f, C, x)=\lim _{\delta \rightarrow 0^{+}} \inf f\left[\mathcal{C}_{f} \cap C \cap(x-\delta, x+\delta)\right]$ and $\overline{\operatorname{LIM}}(f, C, x)=-\underline{\operatorname{LIM}}(-f, C, x)$. The symbols $\underline{\operatorname{LIM}}\left(f, C, x^{-}\right), \underline{\operatorname{LIM}}\left(f, C, x^{+}\right)$, etc., are defined analogously. Moreover let $\underline{\operatorname{LIM}}\left(f, x^{+}\right)=\underline{\operatorname{LIM}}\left(f, \mathbb{R}, x^{+}\right)$, etc. Finally let $\|f\|=\sup |f|[\mathbb{R}]$.

The following are classes of functions.

$\mathbf{B}_{\alpha}$ denotes Baire class $\alpha\left(\alpha<\omega_{1}\right)$.

Key Words: quasi-continuous function, cliquish function, product of functions

Mathematical Reviews subject classification: Primary 28A15. Secondary 26A21, 54C08, $54 \mathrm{C} 30$

Received by the editors April 9, 1997 
$\mathbf{C}_{q}$ consists of all cliquish functions [12]; i.e., $f \in \mathbf{C}_{q}$ if and only if for each open interval $I$ and each $\varepsilon>0$ there is a nondegenerate interval $J \subset I$ with $\omega(f, J)<\varepsilon$. Equivalently, $f \in \mathbf{C}_{q}$ if and only if $f$ is pointwise discontinuous; i.e., if $\mathrm{cl}_{f}=\mathbb{R}$.

D consists of all Darboux functions; i.e., $f \in \mathbf{D}$ if and only if the set $f[J]$ is connected for every interval $J$.

L consists of all Lebesgue measurable functions.

Q consists of all quasi-continuous functions in the sense of Kempisty [6]; i.e., $f \in \mathbf{Q}$ if and only if for each pair of open intervals $I$ and $J$ if $I \cap f^{-1}(J) \neq \emptyset$, then $\operatorname{int}\left(I \cap f^{-1}(J)\right) \neq \emptyset$. Observe that $\mathbf{Q} \subset \mathbf{C}_{q}$.

$\mathbb{R}^{\mathbb{R}}$ consists of all functions.

$\dot{\mathbf{S}}_{s}$ consists of all strong Światkowski functions [7]; i.e., $f \in \dot{\mathbf{S}}_{s}$ if and only if $\mathcal{C}_{f} \cap[f=y] \cap\left(x_{1}, x_{2}\right) \neq \emptyset$ whenever $x_{1}<x_{2}$ and $y$ is a number between $f\left(x_{1}\right)$ and $f\left(x_{2}\right)$. Observe that $\dot{\mathbf{S}}_{s} \subsetneq \mathbf{D} \cap \mathbf{Q}$. (See also Lemma 2.2.)

We omit the intersection sign between classes of functions; e.g., $\mathbf{D B}_{2}$ is the family of all Darboux Baire two functions.

Let $f$ be a function and $x \in \mathbb{R}$. Set $c=\varliminf_{t \rightarrow x^{+}} f(x)$ and $d=\overline{\lim }_{t \rightarrow x^{+}} f(x)$. We say that $x \in \mathbb{R}$ is a Darboux point of $f$ from the right if $c \leq f(x) \leq d$ and $f[(x, x+\delta)] \supset(c, d)$ for each $\delta>0$. Similarly we define the notion of a Darboux point from the left. We say that $x$ is a Darboux point of $f$ if $x$ is a Darboux point of $f$ both from the left and from the right. Recall that $f \in \mathbf{D}$ if and only if each $x \in \mathbb{R}$ is a Darboux point of $f$. (See, e.g., [3, Theorem 5.1].)

\section{Introduction}

It is quite evident that the problem of characterizing of the products of bounded positive (Darboux) quasi-continuous functions can be reduced to the problem of characterizing of the sums of (Darboux) quasi-continuous functions bounded below. In 1995 I studied the class of the sums of nonnegative quasi-continuous functions. Theorem 3.2 generalizes Theorem 7 of [8]. (See also Remark 1.) Its proof is more complicated, however it has the advantage that it can be easily adapted to prove an analogous result concerning sums of nonnegative strong Świątkowski functions (Theorem 3.5).

In Section 4 we deal with products of bounded (Darboux) quasi-continuous functions. (See Theorems 4.3 and 4.6.) The assertion of Theorem 4.6 is valid also for $\mathcal{F}=\mathbf{B}_{1}$, however we have to use different methods to prove it. Therefore this result will be proved elsewhere. 
In Section 5 we study the family of products of strong Świątkowski functions. By Proposition 5.1, this family is essentially smaller than that of products of Darboux quasi-continuous functions. (Recall that in contrast to Proposition 5.1, each cliquish function is the sum of two strong Świattkowski functions [9, Theorem 4].) Propositions 5.2 and 5.3 yield that even if a function $f$ can be written as the product of three strong Świątkowski functions, we cannot conclude that $f$ is the product of two strong Świątkowski functions. Moreover there is a bounded Darboux quasi-continuous function which is discontinuous at a single point, which is the product of a continuous function and a strong Świątkowski one and which cannot be written as a finite product of bounded strong Świạtkowski functions (Corollary 5.5). The problem of characterizing of the family of products of (bounded) strong Świątkowski functions is open.

In the proofs of the main results we will need a few lemmas. Lemma 2.1 is evident and Lemma 2.2 follows by [5, Lemma 2] and Lemma 2.1.

Lemma 2.1. Let $f \in \mathbf{C}_{q}$. Then for each $x \in \mathbb{R}$ and each meager set $A \subset \mathbb{R}$ we have $\underline{\operatorname{LIM}}(f, x)=\underline{\operatorname{LIM}}(f, \mathbb{R} \backslash A, x)$ and $\underline{\operatorname{LIM}}\left(f, x^{-}\right)=\underline{\operatorname{LIM}}\left(f, \mathbb{R} \backslash A, x^{-}\right)$.

Lemma 2.2. Let $f \in \mathbf{C}_{q}$. The following are equivalent:

(i) $f \in \mathbf{Q}$;

(ii) $\underline{\operatorname{LIM}}(|f-f(x)|, \mathbb{R} \backslash A, x)=0$ for each $x \in \mathbb{R}$ and each meager set $A \subset \mathbb{R}$;

(iii) $\underline{\underline{\operatorname{LIM}}}(|f-f(x)|, x)=0$ for each $x \in \mathbb{R}$.

For Darboux quasi-continuous functions we can prove a similar result. (Also see [11, Lemma 2].)

Lemma 2.3. Let $f \in \mathbf{D Q}$. Then for each $x \in \mathbb{R}$ and each meager set $A \subset \mathbb{R}$ we have $\underline{\operatorname{LIM}}\left(|f-f(x)|, \mathbb{R} \backslash A, x^{-}\right)=\underline{\operatorname{LIM}}\left(|f-f(x)|, \mathbb{R} \backslash A, x^{+}\right)=0$.

Lemma 2.4. Let $k \in \mathbb{N}, J=\left[a_{1}, a_{2}\right]$ and let $u: J \rightarrow[0, \infty)$ be continuous. Moreover let $y_{1 j}, \ldots, y_{k j} \geq 0$ be such that $y_{1 j}+\cdots+y_{k j}=u\left(a_{j}\right)(j \in\{1,2\})$. Then there are nonnegative continuous functions $g_{1}, \ldots, g_{k}: J \rightarrow \mathbb{R}$ such that $g_{1}+\cdots+g_{k}=u$ on $J$ and for each $i g_{i}\left(a_{j}\right)=y_{i j}$ for $j \in\{1,2\}$ and $\omega\left(g_{i}, J\right) \leq$ $3 \omega(u, J)+\left|y_{i 2}-y_{i 1}\right|$.

Proof. For each $i$ and for $j \in\{1,2\}$ set

$$
\bar{y}_{i j}= \begin{cases}y_{i j} / u\left(a_{j}\right) & \text { if } u\left(a_{j}\right)>0 \\ k^{-1} & \text { otherwise }\end{cases}
$$


Let $\varphi_{i}: J \rightarrow \mathbb{R}$ be a linear function such that $\varphi_{i}\left(a_{j}\right)=\bar{y}_{i j}$ for $j \in\{1,2\}$. Define $g_{i}=u \varphi_{i}$. Put $c=\min u[J]$ and $d=\max u[J]$. If $d \geq 2 c$, then

$$
\omega\left(g_{i}, J\right) \leq d \max \varphi_{i}[J]-c \min \varphi_{i}[J] \leq d \leq 2(d-c)=2 \omega(u, J) .
$$

Otherwise observe that $u \geq c>d / 2 \geq 0$ on $J$. Assume that $\bar{y}_{i 1} \leq \bar{y}_{i 2}$. (The other case is analogous.) Then

$$
\max g_{i}[J] \leq d \max \varphi_{i}[J]=d \bar{y}_{i 2}=d y_{i 2} / u\left(a_{2}\right) \leq d y_{i 2} / c,
$$

and similarly $\min g_{i}[J] \geq c y_{i 1} / d$. Hence

$$
\begin{aligned}
\omega\left(g_{i}, J\right) & \leq d y_{i 2} / c-c y_{i 1} / d=(d / c+1)(1-c / d) y_{i 2}+\left(y_{i 2}-y_{i 1}\right) c / d \\
& \leq 3(d-c) y_{i 2} / d+\left|y_{i 2}-y_{i 1}\right| \leq 3 \omega(u, J)+\left|y_{i 2}-y_{i 1}\right| .
\end{aligned}
$$

The other requirements of the lemma are evident.

\section{Sums of Nonnegative Functions}

\subsection{Quasi-Continuous Functions}

For brevity, for each $k>1$ the symbol $\mathcal{S}_{k}$ will denote the family of all nonnegative functions $f \in \mathbf{C}_{q}$ such that $\overline{\operatorname{LIM}}(f, x) \geq f(x) / k$ for each $x \in \mathbb{R}$. The next lemma follows from the proof of [8, Lemma 4].

Lemma 3.1. Let $k>1, g \in \mathcal{S}_{k}$ and $\tau>0$. There exists a function $\bar{g} \in \mathcal{S}_{k}$ such that $\mathcal{D}_{\bar{g}} \subset A \stackrel{\text { df }}{=}\left\{x \in \mathbb{R}: \omega(g, x) \geq \tau\left(1-k^{-1}\right)\right\}, g=\bar{g}$ on $A,\|g-\bar{g}\| \leq \tau$ and $g-\bar{g} \in \mathcal{S}_{k}$.

Theorem 3.2. Let $k>1$. For each function $f$ the following are equivalent:

(i) $f$ is the sum of $k$ nonnegative quasi-continuous functions;

(ii) $f \in \mathcal{S}_{k}$;

(iii) there exist functions $\varphi_{1}, \ldots, \varphi_{k} \in \mathbf{B}_{1}$ such that $\varphi_{1}+\cdots+\varphi_{k}=0$ on $\mathbb{R}$ and for $i \in\{1, \ldots, k\}$ the function $f / k+\varphi_{i}$ is nonnegative and quasicontinuous and $\mathcal{D}_{\varphi_{i}} \subset \mathcal{D}_{f}$.

Proof. The implication (iii) $\Rightarrow$ (i) is obvious and the implication (i) $\Rightarrow$ (ii) follows by [8, Theorem 7].

(ii) $\Rightarrow$ (iii). Let $\tau_{0}=\infty$ and $A_{0}=\emptyset$. For each $n \in \mathbb{N}$ set $\tau_{n}=2^{-n}$, define $A_{n}=\left\{x \in \mathbb{R}: \omega(f, x) \geq \tau_{n}\left(1-k^{-1}\right)\right\}$ and observe that $A_{n}$ is a nowhere dense 
closed set. (Recall that the function $\omega(f, \cdot)$ is upper semicontinuous.) We proceed by induction. Put $f_{0}=g_{1,0}=\cdots=g_{k, 0}=0$ and $F_{1,0}=\cdots=F_{k, 0}=\emptyset$. Let $n \in \mathbb{N}$. Assume that for each $j<n$ we have already constructed functions $f_{j}, g_{1, j}, \ldots, g_{k, j}$ and pairwise disjoint nowhere dense sets $F_{1, j}, \ldots, F_{k, j} \subset \mathcal{C}_{f}$ such that

$\left(\mathrm{a}_{j}\right) f_{j}, f-\left(f_{0}+\cdots+f_{j}\right) \in \mathcal{S}_{k}$

$\left(\mathrm{b}_{j}\right) \mathcal{D}_{f_{j}} \subset A_{j}$,

$\left(\mathrm{c}_{j}\right) f=f_{0}+\cdots+f_{j}$ on $A_{j}$,

$\left(\mathrm{d}_{j}\right)\left\|f-\left(f_{0}+\cdots+f_{j}\right)\right\| \leq \tau_{j}$

$\left(\mathrm{e}_{j}\right) \operatorname{cl} F_{i, j}=F_{i, j} \cup A_{j}$ for each $i$,

$\left(\mathrm{f}_{j}\right) \bigcup_{i=1}^{k} \mathcal{D}_{g_{i, j}} \subset A_{j}$,

$\left(\mathrm{g}_{j}\right) g_{1, j}+\cdots+g_{k, j}=f_{0}+\cdots+f_{j}$ on $\mathbb{R}$,

$\left(\mathrm{h}_{j}\right)$ if $j>0$, then $\varphi_{i, j} \stackrel{\mathrm{df}}{=} g_{i, j}-g_{i, j-1}-f_{j} / k \in \mathbf{B}_{1}$ for each $i$.

First find a function $f_{n} \in \mathcal{S}_{k}$ such that conditions $\left(\mathrm{a}_{n}\right)-\left(\mathrm{d}_{n}\right)$ are fulfilled. (We use condition $\left(\mathrm{a}_{n-1}\right)$; cf. Lemma 3.1.) Write $\mathbb{R} \backslash A_{n}$ as the union of a family, $\left\{I_{n, m}: m \in \mathbb{N}\right\}$, consisting of nonoverlapping closed intervals, such that for each $x \in \mathbb{R} \backslash A_{n}$ there are $p, r \in \mathbb{N}$ with $x \in \operatorname{int}\left(I_{n, p} \cup I_{n, r}\right)$. Without loss of generality we may assume that $\lambda\left(I_{n, m}\right)<\varrho\left(I_{n, m}, A_{n}\right)$ and $\omega\left(g_{i, n-1}, I_{n, m}\right)<\tau_{n}$ for each $m$ and $i$ and that $\bigcup_{m \in \mathbb{N}}$ bd $I_{n, m} \subset \mathcal{C}_{f} \backslash \bigcup_{i=1}^{k} F_{i, n-1}$. Fix an $m \in \mathbb{N}$. For each $i$ find a nonempty perfect null-set $F_{i, n, m} \subset \mathcal{C}_{f} \cap \operatorname{int} I_{n, m} \backslash \bigcup_{j=1}^{k} F_{j, n-1}$ such that

$$
g_{i, n-1}+f_{n}>\max \left(g_{i, n-1}+f_{n}\right)\left[I_{n, m}\right]-\tau_{n} / 2^{m}
$$

on $F_{i, n, m}$. Clearly we may assume that the sets $F_{1, n, m}, \ldots, F_{k, n, m}$ are pairwise disjoint.

Fix an $i \in\{1, \ldots, k\}$. Set $F_{i, n}=F_{i, n-1} \cup \bigcup_{m \in \mathbb{N}} F_{i, n, m}$. To prove condition $\left(\mathrm{e}_{n}\right)$ take an arbitrary convergent sequence $\left(x_{s}\right) \subset F_{i, n}$. Suppose that $x_{0}=\lim _{s \rightarrow \infty} x_{s} \notin A_{n}$. If $x_{s} \in F_{i, n-1}$ for infinitely many $s$, then by $\left(\mathrm{e}_{n-1}\right)$

$$
x_{0} \in \operatorname{cl} F_{i, n-1}=F_{i, n-1} \cup A_{n-1} \subset F_{i, n} \cup A_{n} .
$$

Otherwise there is an $m_{0} \in \mathbb{N}$ such that $x_{s} \in F_{i, n, m_{0}}$ for infinitely many $s$. (Recall that each point in $\mathbb{R} \backslash A_{n}$ belongs to $\operatorname{int}\left(I_{n, p} \cup I_{n, r}\right)$ for some $p, r \in \mathbb{N}$.) Then $x_{0} \in F_{i, n, m_{0}} \subset F_{i, n}$. 
We have proved condition $\left(\mathrm{e}_{n}\right)$. Clearly, by the construction, $F_{i, n}$ is a nullset of the first category and the condition $\left(\mathrm{e}_{n}\right)$ implies that $F_{i, n}$ is a nowhere dense set.

Let $G_{n} \supset \bigcup_{i=1}^{k} F_{i, n}$ be an open subset of $\bigcup_{m \in \mathbb{N}}$ int $I_{n, m}$. (See Remark 1 for explanation why we do not set $G_{n}=\bigcup_{m \in \mathbb{N}}$ int $I_{n, m}$.) Notice that the set $U_{n}=G_{n} \backslash \bigcup_{i=1}^{k} F_{i, n}$ is open. For each $i$ and $m$ let $\psi_{i, n, m}$ be a continuous surjection from $F_{i, n, m}$ onto

$$
\left[\max \left\{\min g_{i, n-1}\left[I_{n, m}\right]-\tau_{n-1}, 0\right\}, \max \left\{\max \left(g_{i, n-1}+f_{n}\right)\left[I_{n, m}\right]-\tau_{n} / 2^{m}, 0\right\}\right] .
$$

For each $i$ define the function $g_{i, n}$ on $\mathbb{R} \backslash U_{n}$ by

$$
g_{i, n}(x)= \begin{cases}g_{i, n-1}(x) & \text { if } x \in F_{i, n-1}, \\ \left(g_{i, n-1}+f_{n} /(k-1)\right)(x) & \text { if } x \in F_{j, n-1}, j \neq i, \\ \psi_{i, n, m}(x) & \text { if } x \in F_{i, n, m}, m \in \mathbb{N}, \\ \left(g_{i, n-1}+\frac{g_{j, n-1}+f_{n}-\psi_{j, n, m}}{k-1}\right)(x) & \text { if } x \in F_{j, n, m}, j \neq i, m \in \mathbb{N}, \\ \left(g_{i, n-1}+f_{n} / k\right)(x) & \text { if } x \in \mathbb{R} \backslash G_{n} .\end{cases}
$$

One can easily show that $g_{1, n}+\cdots+g_{k, n}=g_{1, n-1}+\cdots+g_{k, n-1}+f_{n}$ on $\mathbb{R} \backslash U_{n}$ and for each $i: g_{i, n}$ is continuous on $\mathbb{R} \backslash\left(U_{n} \cup A_{n}\right), g_{i, n} \geq 0$ on $\mathbb{R} \backslash U_{n}$ and

$$
\left|g_{i, n}-g_{i, n-1}-f_{n} / k\right|<3 \tau_{n-1} \text { on } \mathbb{R} \backslash U_{n} .
$$

(Notice that $\left\|f_{n}\right\| \leq\left\|f-\left(f_{0}+\cdots+f_{n-1}\right)\right\| \leq \tau_{n-1}$.)

Let $\left(a_{1}, a_{2}\right)$ be a component of $U_{n}$ and $J=\left[a_{1}, a_{2}\right]$. There is an $m \in \mathbb{N}$ such that $J \subset I_{n, m}$. By $\left(\mathrm{b}_{n}\right)$ and $\left(\mathrm{f}_{n-1}\right)$, we have $J \subset \mathcal{C}_{f_{n}} \cap \bigcap_{i=1}^{k} \mathcal{C}_{g_{i, n-1}}$. Use Lemma 2.4 to extend functions $g_{1, n}, \ldots, g_{k, n}$ on $J$ so that $g_{1, n}+\cdots+g_{k, n}=$ $g_{1, n-1}+\cdots+g_{k, n-1}+f_{n}$ on $J$ and for each $i$ : $g_{i, n}\lceil J$ is nonnegative and continuous and

$$
\omega\left(g_{i, n}, J\right) \leq 3 \omega\left(g_{1, n-1}+\cdots+g_{k, n-1}+f_{n}, J\right)+\left|g_{i, n}\left(a_{2}\right)-g_{i, n}\left(a_{1}\right)\right| .
$$

Observe that by (1) and (2), for each $i \in\{1, \ldots, k\}$ and each $x \in J$ we have

$$
\begin{aligned}
& \left|\left(g_{i, n}-g_{i, n-1}-f_{n} / k\right)(x)\right|<\omega\left(g_{i, n}, J\right)+\omega\left(g_{i, n-1}+f_{n} / k, J\right)+3 \tau_{n-1} \\
& \quad \leq 3\left(\sum_{j=1}^{k} \omega\left(g_{j, n-1}, I_{n, m}\right)+\left\|f_{n}\right\|\right)+\left(2 \cdot 3 \tau_{n-1}+\omega\left(g_{i, n-1}+f_{n} / k, J\right)\right) \\
& \quad+\omega\left(g_{i, n-1}+f_{n} / k, J\right)+3 \tau_{n-1} \\
& \leq 3\left(k \tau_{n}+\tau_{n-1}\right)+6 \tau_{n-1}+2\left(\tau_{n}+\tau_{n-1} / k\right)+3 \tau_{n-1} \leq(3 k / 2+14) \tau_{n-1} .
\end{aligned}
$$


In this way we extended functions $g_{1, n}, \ldots, g_{k, n}$ to entire real line. Conditions $\left(g_{n}\right)$ and $\left(h_{n}\right)$ are evident and condition $\left(f_{n}\right)$ follows by $(2)$. This completes the induction step.

Fix an $i \in\{1, \ldots, k\}$. Define $\varphi_{i}=\sum_{n \in \mathbb{N}} \varphi_{i, n}$ and $g_{i}=f / k+\varphi_{i}$. The series is uniformly convergent; so $\varphi_{i} \in \mathbf{B}_{1}$ and $\mathcal{D}_{\varphi_{i}} \subset \bigcup_{n \in \mathbb{N}} A_{n}=\mathcal{D}_{f}$. Moreover

$$
g_{i}=\sum_{n \in \mathbb{N}}\left(f_{n} / k+\varphi_{i, n}\right)=\lim _{s \rightarrow \infty} \sum_{n=1}^{s}\left(g_{i, n}-g_{i, n-1}\right)=\lim _{s \rightarrow \infty} g_{i, s} .
$$

Hence $g_{i} \geq 0$ and $g_{1}+\cdots+g_{k}=f$ on $\mathbb{R}$. Consequently, $\varphi_{1}+\cdots+\varphi_{k}=0$ on $\mathbb{R}$.

To complete the proof we will show that $g_{i} \in \mathbf{Q}$. Let $l \in \mathbb{N}$ and $x \in \mathcal{D}_{g_{i}}$. Then $x \in A_{n} \backslash A_{n-1}$ for some $n \in \mathbb{N}$ and $g_{i}(x)=g_{i, n}(x)=\left(g_{i, n-1}+f_{n} / k\right)(x)$. Since $x \in \mathcal{C}_{g_{i, n-1}}$, there is a $\delta \in\left(0, l^{-1}\right)$ such that $\left|g_{i, n-1}-g_{i, n-1}(x)\right|<2^{-l}$ on $(x-\delta, x+\delta)$. We may assume that $\varrho\left(x, I_{n, m}\right)>\delta$ for each $m<l$. There exists a $t \in \mathcal{C}_{f} \cap\left[f_{n} \geq f_{n}(x) / k-2^{-l}\right] \cap(x-\delta, x+\delta)$. (We use the definition of the class $\mathcal{S}_{k}$ and Lemma 2.1.) Then $t \in I_{n, m}$ for some $m \geq l$. So we can find an $x_{l} \in F_{i, n, m}$ such that $\left|\psi_{i, n, m}\left(x_{l}\right)-\left(g_{i, n-1}+f_{n} / k\right)(x)\right| \leq 3 \cdot 2^{-l}$. Consequently, $x_{l} \in \mathcal{C}_{g_{i}} \cap\left[\left|g_{i}-g_{i}(x)\right| \leq 3 \cdot 2^{-l}\right] \cap\left(x-2 l^{-1}, x+2 l^{-1}\right)$. Вy Lemma 2.2, we conclude that $g_{i} \in \mathbf{Q}$.

Remark 1. In the above proof, one can easily show that $\left|\varphi_{i, n}\right| \leq \sum_{s=1}^{n} f_{s}$ for each $i$ and $n$. Thus if we require $\left\|\sum_{s=1}^{n} f_{s} \cdot \chi_{I_{n, m}}\right\| \cdot \lambda\left(G_{n} \cap I_{n, m}\right)<2^{-m} \lambda\left(I_{n, m}\right)$ for each $n$ and $m$, then we will obtain that each $\varphi_{i, n}$ is a Lebesgue function. (Recall that $\psi$ is a Lebesgue function if $\lim _{\delta \rightarrow 0} \int_{x}^{x+\delta} \delta^{-1}|\psi-\psi(x)|=0$ for each $x \in \mathbb{R}$.) Hence each $\varphi_{i}$ is Lebesgue as well. Since Lebesgue functions are both approximately continuous and derivatives, the answer to both questions asked in [8, p. 157] is affirmative.

Remark 2. Let $\tau>0$ and let $I$ be a nondegenerate interval. In the above proof, if for $n \geq 1$ we put $\tau_{n}=\tau / 2^{n}$ and $A_{n}=\left\{x \in \mathbb{R}: \omega(f, x) \geq \tau_{n}(1-\right.$ $\left.\left.k^{-1}\right)\right\} \cup \operatorname{bd} I$, then for each $i$ we will have $g_{i}\left[\mathcal{C}_{g_{i}} \cap I\right] \supset\left[0, \max \left\{\sup f\left[\mathcal{C}_{f} \cap I\right]-\right.\right.$ $3 \tau / 2,0\}], g_{i}=f / k$ on bd $I$ and $\mathcal{D}_{g_{i}} \subset \mathcal{D}_{f} \cup \operatorname{bd} I$.

Corollary 3.3. Let $k>1$. For each function $f$ the following are equivalent:

(i) $f$ is the sum of $k$ quasi-continuous functions bounded below;

(ii) $f \in \mathbf{C}_{q}, f$ is bounded below and $\inf \{\overline{\operatorname{LIM}}(f, x)-f(x) / k: x \in \mathbb{R}\}>-\infty$;

(iii) there exist functions $\varphi_{1}, \ldots, \varphi_{k} \in \mathbf{B}_{1}$ such that $\varphi_{1}+\cdots+\varphi_{k}=0$ on $\mathbb{R}$ and for $i \in\{1, \ldots, k\}$ the function $f / k+\varphi_{i}$ is bounded below and quasicontinuous and $\mathcal{D}_{\varphi_{i}} \subset \mathcal{D}_{f}$. 
Proof. The implication (iii) $\Rightarrow$ (i) is obvious and the implication (i) $\Rightarrow$ (ii) follows by [8, Corollary 8].

(ii) $\Rightarrow$ (iii). Let $s \leq \inf \left\{\overline{\operatorname{LIM}}(f, x)-f(x) / k: x \in \mathcal{D}_{f}\right\} /(1-1 / k)$ be such that $f \geq s$ on $\mathbb{R}$. Then the function $\widehat{f}=f-s$ is nonnegative and

$$
\overline{\operatorname{LIM}}(\widehat{f}, x)=\overline{\operatorname{LIM}}(f, x)-s \geq f(x) / k-s / k=\widehat{f}(x) / k
$$

for each $x \in \mathcal{D}_{f}$. So by Theorem 3.2, there exist functions $\varphi_{1}, \ldots, \varphi_{k} \in$ $\mathbf{B}_{1}$ such that $\varphi_{1}+\cdots+\varphi_{k}=0$ on $\mathbb{R}$ and for $i \in\{1, \ldots, k\}$ : the function $f / k-s / k+\varphi_{i}$ is nonnegative and quasi-continuous and $\mathcal{D}_{\varphi_{i}} \subset \mathcal{D}_{f}$. Clearly these functions fulfill our requirements.

\subsection{Strong Świạtkowski Functions}

For brevity, for each $k>1$ the symbol $\mathcal{S}_{k}^{*}$ will denote the family of all nonnegative functions $f \in \mathbf{C}_{q}$ such that $\min \left\{\overline{\operatorname{LIM}}\left(f, x^{-}\right), \overline{\operatorname{LIM}}\left(f, x^{+}\right)\right\} \geq f(x) / k$ for each $x \in \mathbb{R}$. The proof of Lemma 3.4 is analogous to that of Lemma 3.1.

Lemma 3.4. Let $k>1, g \in \mathcal{S}_{k}^{*}$ and $\tau>0$. There exists a function $\bar{g} \in \mathcal{S}_{k}^{*}$ such that $\mathcal{D}_{\bar{g}} \subset A \stackrel{\text { df }}{=}\left\{x \in \mathbb{R}: \omega(g, x) \geq \tau\left(1-k^{-1}\right)\right\}, g=\bar{g}$ on $A,\|g-\bar{g}\| \leq \tau$ and $g-\bar{g} \in \mathcal{S}_{k}^{*}$.

Theorem 3.5. Let $k>1$. For each function $f$ the following are equivalent:

(i) $f$ is the sum of $k$ nonnegative Darboux quasi-continuous functions;

(ii) $f \in \mathcal{S}_{k}^{*}$;

(iii) there exist functions $\varphi_{1}, \ldots, \varphi_{k} \in \mathbf{B}_{1}$ such that $\varphi_{1}+\cdots+\varphi_{k}=0$ on $\mathbb{R}$ and for $i \in\{1, \ldots, k\}$ the function $f / k+\varphi_{i}$ is nonnegative and strong Światkowskiand $\mathcal{D}_{\varphi_{i}} \subset \mathcal{D}_{f}$.

Proof. The implication (iii) $\Rightarrow$ (i) is obvious and the implication (i) $\Rightarrow$ (ii) can be proved as in $[8$, Theorem 7$]$.

(ii) $\Rightarrow$ (iii). First we proceed as in the proof of the implication (ii) $\Rightarrow$ (iii) of Theorem 3.2 to construct functions $\varphi_{1}, \ldots, \varphi_{k} \in \mathbf{B}_{1}$. The only difference is to require $f_{1}, f_{2}, \ldots \in \mathcal{S}_{k}^{*}$. (We use Lemma 3.4 instead of Lemma 3.1.) To complete the proof suppose that $g_{i}=f / k+\varphi_{i} \notin \mathbf{S}_{s}$ for some $i \in\{1, \ldots, k\}$. Let $x_{1}, x_{2} \in \mathbb{R}$ and $y \in\left(g_{i}\left(x_{1}\right), g_{i}\left(x_{2}\right)\right)$ be such that $g_{i}(t)=y$ for no $t \in \mathcal{C}_{f} \cap J$, where $J$ is the open interval with end points $x_{1}$ and $x_{2}$. Assume $x_{1}<x_{2}$. (The opposite case is similar.) Set

$$
x_{0}=\sup \left\{x \in\left[x_{1}, x_{2}\right]: g_{i}(t)<y \text { for each } t \in \mathcal{C}_{f} \cap\left(x_{1}, x\right]\right\} .
$$


It is evident that $x_{0} \notin \mathcal{C}_{f}$. Let $n \in \mathbb{N}$ be such that $x_{0} \in A_{n} \backslash A_{n-1}$. Then $g_{i}\left(x_{0}\right)=\left(g_{i, n-1}+f_{n} / k\right)\left(x_{0}\right)$. We consider two cases.

If $\varepsilon=g_{i}\left(x_{0}\right)-y>0$, then $x_{0}>x_{1}$. Choose an $l \in \mathbb{N}$ with $\tau_{n} / 2^{l}<$ $\varepsilon / 3$. Since $x_{0} \in \mathcal{C}_{g_{i, n-1}}, g_{i, n-1}>g_{i, n-1}\left(x_{0}\right)-\varepsilon / 3$ on $\left(x_{0}-\delta, x_{0}\right)$ for some $\delta \in\left(0,\left(x_{0}-x_{1}\right) / 2\right)$. We may assume that $\varrho\left(x, I_{n, m}\right)>\delta$ for $m<l$. By the definition of the class $\mathcal{S}_{k}^{*}$, there is a $t \in \mathcal{C}_{f} \cap\left[f_{n} \geq f_{n}\left(x_{0}\right) / k-\varepsilon / 3\right] \cap\left(x_{0}-\delta, x_{0}\right)$. Then $t \in I_{n, m}$ for some $m \geq l$. Thus

$$
\max \left(g_{i, n-1}+f_{n}\right)\left[I_{n, m}\right]-\tau_{n} / 2^{m}>\left(g_{i, n-1}+f_{n}\right)(t)-\varepsilon / 3>g_{i}\left(x_{0}\right)-\varepsilon=y .
$$

Hence there is a $t_{0} \in F_{i, n, m} \subset \mathcal{C}_{f} \cap\left(x_{1}, x_{0}\right)$ with $g_{i}\left(t_{0}\right)=\psi_{i, n, m}\left(t_{0}\right)>y$, contradicting the definition of $x_{0}$. (Recall that $\lambda\left(I_{n, m}\right)<\varrho\left(I_{n, m}, A_{n}\right)<\delta$.)

If $g_{i}\left(x_{0}\right) \leq y$, then $x_{0}<x_{2}$ and $g_{i, n-1}\left(x_{0}\right) \leq g_{i}\left(x_{0}\right) \leq y$. Since $x_{0} \in \mathcal{C}_{g_{i, n-1}}$, so $g_{i, n-1}<y+\tau_{n-1}$ on $\left(x_{0}, x_{0}+\delta\right)$ for some $\delta \in\left(0,\left(x_{2}-x_{0}\right) / 2\right)$. We will prove by induction that for each $s \geq n$

$$
g_{i, s-1}<y+\tau_{s-1} \quad \text { on } \mathcal{C}_{f} \cap\left(x_{0}, x_{0}+\delta\right) .
$$

This relation clearly holds for $s=n$. Assume it is fulfilled for some $s \geq n$ but not for $s+1$. There is a $t \in \mathcal{C}_{f} \cap\left(x_{0}, x_{0}+\delta\right)$ with $g_{i, s}(t) \geq y+\tau_{s}$. Then $t \in I_{s, m}$ for some $m \in \mathbb{N}$. Let $a=\inf I_{s, m}$. If $g_{i, s}(a) \geq y+\tau_{s} / 2$. Then by inductive assumption, we obtain

$$
\begin{aligned}
\max \left(g_{i, s-1}+f_{s}\right)\left[I_{s, m}\right]-\tau_{s} / 2^{m} & \geq\left(g_{i, s-1}+f_{s} / k\right)(a)-\tau_{s} / 2=g_{i, s}(a)-\tau_{s} / 2 \\
\geq y & \geq g_{i, s-1}(a)-\tau_{s-1} \geq \min g_{i, s-1}\left[I_{s, m}\right]-\tau_{s-1}
\end{aligned}
$$

Hence there is a $t_{0} \in F_{i, s, m} \subset \mathcal{C}_{f} \cap\left(x_{0}, x_{2}\right)$ with $g_{i}\left(t_{0}\right)=\psi_{i, s, m}\left(t_{0}\right)=y$, an impossibility.

Consequently there is an $\hat{a} \in \mathcal{C}_{f} \cap(a, t)$ with $y+\tau_{s} / 2<g_{i, s}(\hat{a})<y+\tau_{s}$. (Recall that $t \in I_{s, m} \subset \mathcal{C}_{g_{i, s}}$ ) Then $\hat{a} \in I_{s+1, p}$ for some $p \in \mathbb{N}$. Thus

$$
\begin{aligned}
\max \left(g_{i, s}+f_{s+1}\right)\left[I_{s+1, p}\right]-\tau_{s+1} / 2^{p} & \geq g_{i, s}(\hat{a})-\tau_{s+1} / 2 \\
>y & >g_{i, s}(\hat{a})-\tau_{s} \geq \min g_{i, s}\left[I_{s+1, p}\right]-\tau_{s} .
\end{aligned}
$$

Hence there is a $t_{0} \in F_{i, s+1, p} \subset \mathcal{C}_{f} \cap\left(x_{0}, x_{2}\right)$ such that $g_{i}\left(t_{0}\right)=\psi_{i, s+1, p}\left(t_{0}\right)=y$, an impossibility. Thus (4) is proved.

By (3) and (4), we conclude that $g_{i}=\lim _{s \rightarrow \infty} g_{i, s} \leq y$ on $\mathcal{C}_{f} \cap\left(x_{0}, x_{0}+\delta\right)$. But by our assumption, $g_{i} \neq y$ on $\mathcal{C}_{f} \cap\left[x_{1}, x_{2}\right]$. Thus $g_{i}<y$ on $\mathcal{C}_{f} \cap\left(x_{1}, x_{0}+\delta\right)$, contradicting the definition of $x_{0}$.

The proof of the next corollary mimics that of Corollary 3.3. (We use Theorem 3.5 instead of Theorem 3.2.) 
Corollary 3.6. Let $k>1$. For each function $f$ the following are equivalent:

(i) $f$ is the sum of $k$ Darboux quasi-continuous functions bounded below;

(ii) $f \in \mathbf{C}_{q}$, $f$ is bounded below and

$$
\inf \left\{\min \left\{\overline{\operatorname{LIM}}\left(f, x^{-}\right), \overline{\operatorname{LIM}}\left(f, x^{+}\right)\right\}-f(x) / k: x \in \mathbb{R}\right\}>-\infty ;
$$

(iii) there exist functions $\varphi_{1}, \ldots, \varphi_{k} \in \mathbf{B}_{1}$ such that $\varphi_{1}+\cdots+\varphi_{k}=0$ on $\mathbb{R}$ and for $i \in\{1, \ldots, k\}$ the function $f / k+\varphi_{i}$ is bounded below and strong Światkowski and $\mathcal{D}_{\varphi_{i}} \subset \mathcal{D}_{f}$.

\section{Products of Bounded Functions}

In this section for brevity we define $\mathfrak{F}=\left\{\mathbb{R}^{\mathbb{R}}, \mathbf{L}\right\} \cup\left\{\mathbf{B}_{\alpha}: \alpha \geq 1\right\}$.

\subsection{Quasi-Continuous Functions}

Lemma 4.1. Suppose $\mathcal{F} \in \mathfrak{F}, f \in \mathbf{C}_{q} \mathcal{F}$ is bounded, $f>0$ on an interval $I=[a, b), a \in \mathcal{C}_{f}, k>1$ and $T \geq \sqrt[k]{\|f\|}+1$. Assume moreover that

$$
\underline{\operatorname{LIM}}(|f|, x) \leq T^{k-1} \sqrt[k]{|f(x)|}
$$

for each $x \in(a, b)$. Then for each $\varepsilon \in\left(\inf f\left[\mathcal{C}_{f} \cap I\right], T\right]$ there are positive functions $g_{1}, \ldots, g_{k} \in \mathcal{F}$ such that $f=g_{1} \ldots g_{k}$ on $I$ and for each $i, g_{i}\lceil I$ is quasi-continuous, $\left\|g_{i}\right\| \leq T$ and $g_{i}\left[\mathcal{C}_{g_{i}} \cap I\right] \supset\left[\varepsilon / T^{k-1}, T\right]$.

Proof. Define $\widehat{f}(x)=k \ln T-\ln f(x)$ if $x \in I$ and $\widehat{f}(x)=0$ otherwise. Then $\widehat{f}$ is nonnegative and cliquish, $\widehat{f}$ is at least unilaterally continuous on $(-\infty, a]$ and $[b, \infty)$ and by $(5)$, for each $x \in(a, b)$

$$
\overline{\operatorname{LIM}}(\widehat{f}, x)=k \ln T-\ln \underline{\operatorname{LIM}}(f, x) \geq \ln T-k^{-1} \ln f(x)=\widehat{f}(x) / k .
$$

Thus $\widehat{f} \in \mathcal{S}_{k}$. By Remark 2, there are nonnegative functions $\widehat{g}_{1}, \ldots, \widehat{g}_{k} \in \mathbf{Q} \mathcal{F}$ such that $\widehat{f}=\widehat{g}_{1}+\cdots+\widehat{g}_{k}$ on $\mathbb{R}$ and for each $i: \widehat{g}_{i}\left[\mathcal{C}_{\widehat{g}_{i}} \cap I\right] \supset\left[0, \ln \left(T^{k} / \varepsilon\right)\right]$ and $\widehat{g}_{i}(a)=\widehat{f}(a) / k$. For each $i$ define $g_{i}=T \cdot \chi_{I} / \exp \circ \widehat{g}_{i}$. Then clearly $f=g_{1} \ldots g_{k}$ on $I$. Let $i \in\{1, \ldots, k\}$. Since $\widehat{g}_{i}=0$ on $(-\infty, a)$ and $\widehat{g}_{i}(a)>0$, so $\widehat{g}_{i}$ is not quasi-continuous from the left at $a$. Hence $g_{i}\lceil I$ is quasi-continuous. Moreover $0<g_{i} \leq T$ on $I$ and $g_{i}\left[\mathcal{C}_{g_{i}} \cap I\right] \supset\left[\varepsilon / T^{k-1}, T\right]$. 
Lemma 4.2. Suppose $\mathcal{F} \in \mathfrak{F}, f \in \mathbf{C}_{q} \mathcal{F}$ is bounded, $f$ is either positive or negative on an interval $I=[a, b), a \in \mathcal{C}_{f}, k>1$ and $T \geq \sqrt[k]{\|f\|}+1$. Assume moreover that condition (5) holds for each $x \in(a, b)$. Then for each $\varepsilon \in\left(\inf |f|\left[\mathcal{C}_{f} \cap I\right], T\right]$ there are functions $g_{1}, \ldots, g_{k} \in \mathcal{F}$ such that $f=g_{1} \ldots g_{k}$ on $I$ and for each $i, g_{i}\left\lceil I\right.$ is quasi-continuous, $\left\|g_{i}\right\| \leq T$ and

$$
g_{i}\left[\mathcal{C}_{g_{i}} \cap I\right] \supset\left[-T,-\varepsilon / T^{k-1}\right] \cup\left[\varepsilon / T^{k-1}, T\right] .
$$

Proof. Choose $x_{1}, x_{2} \in \mathcal{C}_{f} \cap[|f|<\varepsilon] \cap(a, b)$ with $x_{1}<x_{2}$. Set $I_{1}=\left[a, x_{1}\right)$, $I_{2}=\left[x_{1}, x_{2}\right)$ and $I_{3}=\left[x_{2}, b\right)$. For $j \in\{1,2,3\}$ use Lemma 4.1 to construct positive functions $g_{1 j}, \ldots, g_{k j} \in \mathcal{F}$ such that $|f|=g_{1 j} \ldots g_{k j}$ on $I_{j}$ and for each $i g_{i j} \mid I_{j}$ is quasi-continuous, $\left\|g_{i j}\right\| \leq T$ and $g_{i j}\left[\mathcal{C}_{g_{i j}} \cap I_{j}\right] \supset\left[\varepsilon / T^{k-1}, T\right]$. Define $\left\{t_{i j}: i \in\{1, \ldots, k\}, j \in\{1,2,3\}\right\}$ so that $t_{1 j} \ldots t_{k j}=\operatorname{sgn} f[I]$ for each $j$ and $\left\{t_{i j}: j \in\{1,2,3\}\right\}=\{-1,1\}$ for each $i$. For each $i \in\{1, \ldots, k\}$ put $g_{i}=\sum_{j=1}^{3} t_{i j} \cdot g_{i j} \cdot \chi_{I_{j}}$. One can easily see that then the requirements of the lemma are fulfilled.

Theorem 4.3. Suppose that $\mathcal{F} \in \mathfrak{F}$ and $k>1$. For each function $f \in \mathcal{F}$ the following are equivalent:

(i) $f$ is the product of $k$ bounded quasi-continuous functions;

(ii) $f \in \mathbf{C}_{q}, f$ is bounded, the set $[f=0]$ is simply open and there exists a $T \geq \sqrt[k]{\|f\|}+1$ such that condition (5) holds for each $x \in \mathbb{R}$;

(iii) there exist bounded functions $g_{1}, \ldots, g_{k} \in \mathbf{Q} \mathcal{F}$ such that $f=g_{1} \ldots g_{k}$ on $\mathbb{R}$.

Proof. The implication (iii) $\Rightarrow$ (i) is obvious.

(i) $\Rightarrow$ (ii). Let $f=g_{1} \ldots g_{k}$, where $g_{1}, \ldots, g_{k} \in \mathbf{Q}$ are bounded. The boundedness and cliquishness of $f$ are obvious and the set $[f=0]$ is simply open by [10]. Put $T=\max \left\{\left\|g_{i}\right\|: i \in\{1, \ldots, k\}\right\}+1$ and fix an $x \in \mathbb{R}$. Then $\left|g_{i}(x)\right| \leq \sqrt[k]{|f(x)|}$ for some $i \in\{1, \ldots, k\}$. By Lemma 2.2, there is a sequence $\left(x_{n}\right) \subset \mathfrak{e}_{f}$ such that $x_{n} \rightarrow x$ and $g_{i}\left(x_{n}\right) \rightarrow g_{i}(x)$. Hence

$$
\underline{\operatorname{LIM}}(|f|, x) \leq \underline{\lim }_{n \rightarrow \infty}\left|f\left(x_{n}\right)\right| \leq \prod_{j \neq i}\left\|g_{j}\right\| \cdot \lim _{n \rightarrow \infty}\left|g_{i}\left(x_{n}\right)\right| \leq T^{k-1} \sqrt[k]{|f(x)|} .
$$

(ii) $\Rightarrow$ (iii). Let $G_{0}=\operatorname{int}[f=0]$ and $G_{1}=\operatorname{int}[f<0] \cup \operatorname{int}[f>0]$. For $p \in\{0,1\}$ write $G_{p}$ as the union of a family, $\left\{\left[a_{p n}, b_{p n}\right): n \in \mathbb{N}\right\}$, consisting of pairwise disjoint intervals, such that $\left\{a_{p n}: n \in \mathbb{N}\right\} \subset \mathcal{C}_{f}$. For each $n \in \mathbb{N}$ construct continuous functions $g_{10 n}, \ldots, g_{k 0 n}$ such that $g_{10 n} \ldots g_{k 0 n}=0$ on $\mathbb{R}$ and $g_{10 n}\left[\left[a_{0 n}, b_{0 n}\right)\right]=\cdots=g_{k 0 n}\left[\left[a_{0 n}, b_{0 n}\right)\right]=[-T, T]$ and find functions 
$g_{11 n}, \ldots, g_{k 1 n} \in \mathcal{F}$ such that $f=g_{11 n} \ldots g_{k 1 n}$ on $\left[a_{1 n}, b_{1 n}\right)$ and for each $i$ : $g_{i 1 n}\left\lceil\left[a_{1 n}, b_{1 n}\right)\right.$ is quasi-continuous, $\left\|g_{i 1 n}\right\| \leq T$ and

$$
g_{i 1 n}\left[\mathcal{C}_{g_{i 1 n}} \cap\left[a_{1 n}, b_{1 n}\right)\right] \supset\left[-T,-\varepsilon_{n} / T^{k-1}\right] \cup\left[\varepsilon_{n} / T^{k-1}, T\right],
$$

where $\varepsilon_{n}=\min \left\{\inf |f|\left[\mathcal{C}_{f} \cap\left[a_{1 n}, b_{1 n}\right)\right]+n^{-1}, T\right\}$. (Cf. Lemma 4.2.)

Fix an $i \in\{1, \ldots, k\}$ and define

$g_{i}(x)= \begin{cases}g_{i p n}(x) & \text { if } x \in\left[a_{p n}, b_{p n}\right) \text { for some } p \in\{0,1\} \\ \sqrt[k]{|f(x)|} \cdot(\operatorname{sgn} f(x))^{1+\operatorname{sgn}(k-i)} & \text { and } n \in \mathbb{N} \\ & \text { otherwise. }\end{cases}$

It is clear that $g_{i}$ is quasi-continuous on $G=G_{0} \cup G_{1}$ and $\left\|g_{i}\right\| \leq T$. Since the set $[f=0]$ is simply open, so $G$ is dense in $\mathbb{R}[2$, Remark 2]. It is easy to prove that for each $x \notin G$ and $y \in\left[-T,-\underline{\operatorname{LIM}}(|f|, x) / T^{k-1}\right] \cup\left[\underline{\operatorname{LIM}}(|f|, x) / T^{k-1}, T\right]$ there is a sequence $\left(x_{n}\right) \subset \mathcal{C}_{g_{i}}$ such that $x_{n} \rightarrow x$ and $g_{i}\left(x_{n}\right) \rightarrow y$. So by condition (5) and Lemma 2.2, we obtain $g_{i} \in \mathbf{Q}$. The other requirements are evident.

\subsection{Darboux Quasi-Continuous Functions}

Lemma 4.4. Let $A \subset \mathbb{R}$ be a nowhere dense closed set, let $\mathcal{J}$ be the family of all components of $\mathbb{R} \backslash A$ and let $f$ be a nonnegative cliquish function such that for each $x \in A$, if $x$ is not isolated in $A$ from the left (from the right), then $\underline{\operatorname{LIM}}\left(f, x^{-}\right)=0$ (respectively $\left.\underline{\operatorname{LIM}}\left(f, x^{+}\right)=0\right)$. There exist pairwise disjoint families $\mathcal{J}_{1}, \ldots, \mathcal{J}_{4} \subset \mathcal{J}$ such that for each $j \in\{1, \ldots, 4\}$ and each $x \in A$, if $x$ is not isolated in A from the left (from the right), then $\underline{\operatorname{LIM}}\left(f, \cup_{\mathcal{J}}, x^{-}\right)=0$ (respectively $\left.\underline{\operatorname{LIM}}\left(f, \bigcup_{\mathcal{J}}, x^{+}\right)=0\right)$.

Proof. I. First suppose $A$ is nonempty, compact and perfect. Then for each locally finite family $\overline{\mathcal{J}} \subset \mathcal{J}$ and each open interval $J$, if $J \cap A \neq \emptyset$, then

$$
\inf \left\{\inf f\left[\mathcal{C}_{f} \cap I\right]: I \in \mathcal{J} \backslash \overline{\mathcal{J}}, I \subset J\right\}=0 .
$$

Set $\mathcal{J}_{1,0}=\cdots=\mathcal{J}_{4,0}=\emptyset$. Let $n \in \mathbb{N}$. Suppose families $\mathcal{J}_{1, n-1}, \ldots, \mathcal{J}_{4, n-1} \subset \mathcal{J}$ are pairwise disjoint and if $n>1$, then

for each $j \in\{1, \ldots, 4\}$ and each $x \in A$ there is an interval $I \in \mathcal{J}_{j, n-1}$
such that $I \subset\left(x-(n-1)^{-1}, x+(n-1)^{-1}\right)$ and inf $f\left[\mathcal{C}_{f} \cap I\right]<(n-1)^{-1}$.

Let $\mathcal{J}_{n}$ be a locally finite cover of $A$ consisting of pairwise disjoint open intervals of diameter less than $n^{-1}$, such that $A \cap J \neq \emptyset$ for each $J \in \mathcal{J}_{n}$. For each $J \in \mathcal{J}_{n}$ use condition (6) to choose intervals $I_{1, J}, \ldots, I_{4, J} \in \mathcal{J} \backslash\left(\mathcal{J}_{1, n-1} \cup \cdots \cup \mathcal{J}_{4, n-1}\right)$ 
such that inf $f\left[\mathcal{C}_{f} \cap I_{j, J}\right]<n^{-1}$ for each $j$. Define $\mathcal{J}_{j, n}=\mathcal{J}_{j, n-1} \cup\left\{I_{j, J}: J \in \mathcal{J}_{n}\right\}$ $(j \in\{1, \ldots, 4\})$. Clearly the families $\mathcal{J}_{1, n}, \ldots, \mathcal{J}_{4, n}$ are pairwise disjoint and fulfill condition $\left(\mathrm{a}_{n}\right)$.

For $j \in\{1, \ldots, 4\}$ put $\mathcal{J}_{j}=\bigcup_{n \in \mathbb{N}} \mathcal{J}_{j, n}$. Clearly these families are pairwise disjoint. To complete the proof of this case fix a $j \in\{1, \ldots, 4\}$ and an $x \in A$ and suppose $x$ is not isolated in $A$ from the left. (The other case is similar.) Fix a $\delta>0$. Let $t \in A \cap(x-\delta / 2, x)$ and let $n>(x-t)^{-1}$. By $\left(\mathrm{a}_{n}\right)$, there is an interval $I \in \mathcal{J}_{j}$ such that $I \subset\left(t-n^{-1}, t+n^{-1}\right) \subset(x-\delta, x)$ and $\inf f\left[\mathfrak{C}_{f} \cap I\right]<n^{-1}<\delta$. Consequently, $\underline{\operatorname{LIM}}\left(f, \cup \mathcal{J}_{j}, x^{-}\right)=0$.

II. Now let $A$ be an arbitrary nowhere dense closed set. Denote by $\mathcal{f}$ the family of all compact intervals, $J$, for which there are pairwise disjoint families $\mathcal{J}_{1}, \ldots, \mathcal{J}_{4} \subset \mathcal{J}$ such that for each $j \in\{1, \ldots, 4\}$ and each $x \in A \cap J$, if $x$ is not isolated in $A \cap J$ from the left (from the right), then $\underline{\operatorname{LIM}}\left(f, \bigcup_{\mathcal{J}_{j}}, x^{-}\right)=0$ (respectively $\left.\underline{\operatorname{LIM}}\left(f, \cup \mathcal{J}_{j}, x^{+}\right)=0\right)$. Moreover let $G$ be the set of all $x \in \mathbb{R}$ for which there exists an interval $\Phi_{x} \in \mathcal{J}$ with $x \in \operatorname{int} \Phi_{x}$.

Claim 1. If $\left[a_{1}, a_{2}\right] \in \mathcal{J}$ and $\left[a_{2}, a_{3}\right] \in \mathcal{J}$, then $\left[a_{1}, a_{3}\right] \in \mathcal{J}$.

For $i \in\{1,2\}$ let $\mathcal{J}_{1, i}, \ldots, \mathcal{J}_{4, i}$ be the families corresponding to $\left[a_{i}, a_{i+1}\right] \in \mathcal{J}$. For $j \in\{1, \ldots, 4\}$ define $\mathcal{J}_{j}=\bigcup_{i=1}^{2}\left\{I \in \mathcal{J}_{j, i}: I \subset\left(a_{i}, a_{i+1}\right)\right\}$. Clearly these families yield $\left[a_{1}, a_{3}\right] \in \mathcal{J}$.

Claim 2. Every compact interval $J \subset G$ belongs to $\mathcal{J}$.

Indeed, the compactness of $J$ and the relation $J \subset \bigcup_{x \in J}$ int $\Phi_{x}$ imply that there are $x_{1}, \ldots, x_{m} \in J$ such that $J \subset \bigcup_{i=1}^{m} \operatorname{int} \Phi_{x_{i}}$. It is clear that if $J \in \mathcal{J}$ and $J^{\prime}$ is a closed subinterval of $J$, then $J^{\prime} \in \mathcal{J}$. Thus we can find nonoverlapping intervals $J_{1}, \ldots, J_{l} \in \mathcal{J}$ with $J=\bigcup_{s=1}^{l} J_{s}$. By Claim 1, we obtain $J \in \mathcal{J}$.

Claim 3. If $a<b$ and $(a, b) \subset G$, then $[a, b] \in \mathcal{J}$.

Let $c \in(a, b)$. It suffices to show that $[a, c] \in \mathcal{J}$ and $[c, b] \in \mathcal{J}$. (See Claim 1.) We will verify only that $[a, c] \in \mathcal{J}$, the proof of the other relation being analogous.

If $A \cap(a, a+\eta)=\emptyset$ for some $\eta \in(0, c-a)$, then let $\mathcal{J}_{1}, \ldots, \mathcal{J}_{4}$ show that $[a+\eta, c] \in \mathcal{J}$. (See Claim 2.) Evidently these families also show that $[a, c] \in \mathcal{J}$ as well.

Otherwise $\inf f\left[\mathcal{C}_{f} \cap(a, a+\eta)\right]=0$ for each $\eta>0$. Set $a_{n}=a+(c-a) / n$ for each $n \in \mathbb{N}$. For each $n$ choose intervals $I_{1, n}, \ldots, I_{4, n} \in \mathcal{J}$ such that $I_{j, n} \subset\left(a, a_{n}\right)$ and $\inf f\left[\mathcal{C}_{f} \cap I_{j, n}\right]<n^{-1}$ for each $j$. By Claim 2, there are 
families $\mathcal{J}_{1, n}, \ldots, \mathcal{J}_{4, n}$ corresponding to $\left[a_{n+1}, a_{n}\right] \in \mathcal{J}$. Notice that we may assume $\bigcup_{j=1}^{4} \bigcup \mathcal{J}_{j, n} \subset\left(a_{n+1}, a_{n}\right)$. For $j \in\{1, \ldots, 4\}$ put

$$
\mathcal{J}_{j}=\left(\left\{I_{j, n}: n \in \mathbb{N}\right\} \cup \bigcup_{n \in \mathbb{N}} \mathcal{J}_{j, n}\right) \backslash\left\{I_{i, n}: i \neq j, n \in \mathbb{N}\right\} .
$$

Clearly these families show that $[a, c] \in \mathcal{J}$.

Claim 4. We have $G=\mathbb{R}$.

By way of contradiction suppose that the set $P=\mathbb{R} \backslash G$ is nonempty. Observe that $P$ is closed. If there are an $x \in P$ and a $\delta>0$ such that $P \cap(x-\delta, x+\delta)=\{x\}$, then by Claim 3 , we obtain $[x-\delta, x] \in \mathcal{J}$ and $[x, x+\delta] \in \mathcal{J}$. Hence by Claim $1,[x-\delta, x+\delta] \in \mathcal{J}$. Thus $x \in G$, an impossibility.

Consequently $P$ is perfect. Let $J$ be a compact interval such that the set $A^{\prime}=P \cap J$ is nonempty and perfect. Since $\mathbb{R} \backslash A \subset G$, so $A^{\prime}$ is nowhere dense. Let $\mathcal{J}^{\prime}=\left\{I_{n}^{\prime}: n \in \mathbb{N}\right\}$ be the family of all components of $\mathbb{R} \backslash A^{\prime}$. By the first part of the proof, there are pairwise disjoint families $J_{1}^{\prime}, \ldots, J_{4}^{\prime} \subset J^{\prime}$ such that for each $j \in\{1, \ldots, 4\}$ and each $x \in A^{\prime}$ if $x$ is not isolated in $A^{\prime}$ from the left (from the right), then $\underline{\operatorname{LIM}}\left(f, \cup \mathcal{J}_{j}^{\prime}, x^{-}\right)=0$ (respectively $\left.\underline{\operatorname{LIM}}\left(f, \cup \mathcal{J}_{j}^{\prime}, x^{+}\right)=0\right)$. Fix an $n \in \mathbb{N}$. If $I_{n}^{\prime}$ is bounded, then let $\mathcal{J}_{1, n}, \ldots, \mathcal{J}_{4, n}$ show that $\mathrm{cl} I_{n}^{\prime} \in \mathcal{J}$ (see Claim 3), otherwise put $\mathcal{J}_{1, n}=\cdots=\mathcal{J}_{4, n}=\emptyset$. Let $\varphi\left(I_{n}^{\prime}\right) \in \mathcal{J}$ be such that $\varphi\left(I_{n}^{\prime}\right) \subset I_{n}^{\prime}$ and $\inf f\left[\mathcal{C}_{f} \cap \varphi\left(I_{n}^{\prime}\right)\right] \leq \inf \left\{\inf f\left[\mathcal{C}_{f} \cap I\right]: I \in \mathcal{J}, I \subset I_{n}^{\prime}\right\}+n^{-1}$.

For $j \in\{1, \ldots, 4\}$ define

$$
\mathcal{J}_{j}=\left(\bigcup_{n \in \mathbb{N}}\left\{I \in \mathcal{J}_{j, n}: I \subset I_{n}^{\prime}\right\} \backslash \varphi\left[\mathcal{J}^{\prime}\right]\right) \cup \varphi\left[\mathcal{J}_{j}^{\prime}\right] .
$$

It is quite evident that these families are pairwise disjoint. Fix a $j \in\{1, \ldots, 4\}$ and an $x \in A \cap J$ and suppose $x$ is not isolated in $A \cap J$ from the left. (The other case is similar.) If $x \in \operatorname{cl} I_{n}^{\prime}$ for some $n \in \mathbb{N}$ and $x$ is not the left end point of $I_{n}^{\prime}$, then

$$
\underline{\operatorname{LIM}}\left(f, \bigcup \mathcal{J}_{j}, x^{-}\right) \leq \underline{\operatorname{LIM}}\left(f, \bigcup \mathcal{J}_{j, n}, x^{-}\right)=0,
$$

and in the opposite case we have

$$
\underline{\operatorname{LIM}}\left(f, \bigcup \mathcal{J}_{j}, x^{-}\right) \leq \underline{\operatorname{LIM}}\left(f, \bigcup \varphi\left[\mathcal{J}_{j}^{\prime}\right], x^{-}\right)=\underline{\operatorname{LIM}}\left(f, \bigcup \mathcal{J}_{j}^{\prime}, x^{-}\right)=0 .
$$

Thus $A^{\prime} \cap \operatorname{int} J \subset P \cap G=\emptyset$. But $A^{\prime} \subset J$ and $A^{\prime}$ is perfect, an impossibility.

Finally for each $z \in \mathbb{Z}$ let $\mathcal{J}_{1, z}, \ldots, \mathcal{J}_{4, z}$ witness $[z, z+1] \in \mathcal{J}$. (See Claims 4 and 2.) For $j \in\{1, \ldots, 4\}$ define $\mathcal{J}_{j}=\bigcup_{z \in \mathbb{Z}}\left\{I \in \mathcal{J}_{j, z}: I \subset(z, z+1)\right\}$. Clearly these families fulfill the requirements of the lemma. 
Lemma 4.5. Suppose $\mathcal{F} \in \mathfrak{F}, f \in \mathbf{C}_{q} \mathcal{F}$ is bounded, $f>0$ on an open interval $I, k>1$ and $T \geq \sqrt[k]{\|f\|}+1$. Assume moreover that $f$ is nonnegative on bd $I$ and that

$$
\max \left\{\underline{\operatorname{LIM}}\left(|f|, x^{-}\right), \underline{\operatorname{LIM}}\left(|f|, x^{+}\right)\right\} \leq T^{k-1} \sqrt[k]{|f(x)|}
$$

for each $x \in \operatorname{cl} I$. Then for each $\varepsilon \in\left(\inf f\left[\mathcal{C}_{f} \cap I\right], T\right]$ there are functions $g_{1}, \ldots, g_{k} \in \mathbf{D Q} \mathcal{F}$ such that $f=g_{1} \ldots g_{k}$ on $I$ and for each $i,\left\|g_{i}\right\| \leq T$, $g_{i}\left[\mathcal{C}_{g_{i}} \cap I\right] \supset\left[\varepsilon / T^{k-1}, T\right]$ and $g_{i}=\sqrt[k]{f}$ on $\operatorname{bd} I$.

Proof. Define $\widehat{f}(x)=k \ln T-\ln f(x)$ if $x \in I$ and $\widehat{f}(x)=0$ otherwise. Then $\widehat{f}$ is nonnegative. By (7), for each $x \in I$

$$
\overline{\operatorname{LIM}}\left(\widehat{f}, x^{-}\right)=k \ln T-\underline{\operatorname{LIM}}\left(\ln f, x^{-}\right) \geq \ln T-k^{-1} \ln f(x)=\widehat{f}(x) / k,
$$

and similarly $\overline{\operatorname{LIM}}\left(\widehat{f}, x^{+}\right) \geq \widehat{f}(x) / k$. But $\widehat{f}=0$ on $\mathbb{R} \backslash I$; so $\widehat{f} \in \mathcal{S}_{k}^{*}$.

Observe that $\eta=\left(\varepsilon-\inf f\left[\mathcal{C}_{f} \cap I\right]\right) / 2>0$. Put $a=\inf I$ and $b=\sup I$ and let $\left\{a_{z}: z \in \mathbb{Z}\right\}$ be a strictly increasing sequence with limit points $a$ and $b$. For each $z \in \mathbb{Z}$ set $I_{z}=\left[a_{z}, a_{z+1}\right]$ and $\eta_{z}=\inf f\left[\mathcal{C}_{f} \cap I_{z}\right]+\eta / 2^{|z|}$ and construct nonnegative functions $\widehat{g}_{1 z}, \ldots, \widehat{g}_{k z} \in \mathbf{D Q} \mathcal{F}$ such that $\widehat{f}=\widehat{g}_{1 z}+\cdots+\widehat{g}_{k z}$ on $\mathbb{R}$ and for each $i$ : $\widehat{g}_{i z}\left[\mathcal{C}_{\widehat{g}_{i z}} \cap I_{z}\right] \supset\left[0, \ln \left(T^{k} / \eta_{z}\right)\right], \widehat{g}_{i z}=\widehat{f} / k$ on $\left\{a_{z}, a_{z+1}\right\}$ and $\mathcal{D}_{\widehat{g}_{i z}} \subset \mathcal{D}_{f} \cup\left\{a_{z}, a_{z+1}\right\}$. (See Remark 2.)

Let $i \in\{1, \ldots, k\}$. Define $g_{i}(x)=T / \exp \left(\widehat{g}_{i z}(x)\right)$ if $x \in I_{z}$ for some $z \in \mathbb{Z}$, $g_{i}(x)=\sqrt[k]{f(x)}$ if $x \in$ bd $I$ and let $g_{i}$ be constant on $(-\infty, a]$ and $[b, \infty)$. It is clear that $g_{i} \in \mathbf{Q} \mathcal{F}$ and that each $x \notin$ bd $I$ is a Darboux point of $g_{i}$. Suppose $a \in \mathbb{R}$. (The case $b \in \mathbb{R}$ is similar.) By construction, for each $c \in I$ we have $g_{i}[(a, c)] \supset\left(\underline{\operatorname{LIM}}\left(f, a^{+}\right) / T^{k-1}, T\right]$. Hence by $(7), a$ is a Darboux point of $g_{i}$ from the right. Thus $g_{i} \in \mathbf{D}$.

Since $\inf f\left[\mathcal{C}_{f} \cap I\right]<\varepsilon$, so $\inf f\left[\mathcal{C}_{f} \cap I_{z}\right]<\inf f\left[\mathcal{C}_{f} \cap I\right]+\eta=\varepsilon-\eta$ for some $z \in \mathbb{Z}$. Then $\eta_{z}<\varepsilon$, whence $g_{i}\left[\mathcal{C}_{g_{i}} \cap I_{z}\right] \supset\left[\varepsilon / T^{k-1}, T\right]$. The other requirements are easy to prove.

Theorem 4.6. Suppose that $\mathcal{F} \in \mathfrak{F} \backslash\left\{\mathbf{B}_{1}\right\}$ and $k>1$. For each function $f \in \mathcal{F}$ the following are equivalent:

(i) $f$ is the product of $k$ bounded Darboux quasi-continuous functions;

(ii) $f \in \mathbf{C}_{q}, f$ is bounded, the set $[f=0]$ is simply open, there exists a $T \geq \sqrt[k]{\|f\|}+1$ such that condition (7) holds for each $x \in \mathbb{R}$ and

$$
\text { for all } x<t \text {, if } f(x) f(t)<0 \text {, then }[f=0] \cap(x, t) \neq \emptyset \text {; }
$$


(iii) there exist bounded functions $g_{1}, \ldots, g_{k} \in \mathbf{D Q} \mathcal{F}$ such that $f=g_{1} \ldots g_{k}$ on $\mathbb{R}$.

Proof. The implication (iii) $\Rightarrow$ (i) is obvious and the implication (i) $\Rightarrow$ (ii) can be proved as in Theorem 4.3. (Recall that condition (8) follows by [4].)

(ii) $\Rightarrow$ (iii). Put $A=\operatorname{bd}(\operatorname{cl}[f=0])$ and let $\mathcal{J}=\left\{I_{n}: n<p\right\}$ be the family of all components of $\mathbb{R} \backslash A$. (Here $p$ is either a positive integer or the infinity.) Observe that by (7), if $x \in A$ and $x$ is not isolated in $A$ from the left (from the right), then $\underline{\operatorname{LIM}}\left(|f|, x^{-}\right)=0$ (respectively $\underline{\operatorname{LIM}}\left(|f|, x^{+}\right)=0$ ). By Lemma 4.4, there are pairwise disjoint families $\mathcal{J}_{1}, \ldots, \mathcal{J}_{4} \subset \mathcal{J}$ such that for each $j \in\{1, \ldots, 4\}$ and each $x \in A$, if $x$ is not isolated in $A$ from the left (from the right), then $\underline{\operatorname{LIM}}\left(|f|, \cup^{\mathcal{I}}, x^{-}\right)=0$ (respectively $\left.\underline{\operatorname{LIM}}\left(|f|, \cup_{\mathcal{J}}, x^{+}\right)=0\right)$. Clearly we may assume $\mathcal{J}=\bigcup_{j=1}^{4} \mathcal{J}_{j}$.

Let $n<p$. If $I_{n}=\left(a_{n}, b_{n}\right) \subset \operatorname{cl}[f=0]$, then $I_{n} \subset[f=0]$. (Recall that the set $[f=0]$ is simply open.) Construct functions $g_{1 n}, \ldots, g_{k n} \in \dot{\mathbf{S}}_{s} \mathbf{B}_{1}$ such that $f=g_{1 n} \ldots g_{k n}$ on $I_{n}$ and $g_{i n}\left[\left(a_{n}, c\right)\right]=g_{i n}\left[\left(c, b_{n}\right)\right]=[-T, T]$ for each $i \in\{1, \ldots, k\}$ and each $c \in I_{n}$. Otherwise by (8), either $f>0$ on $I_{n}$ and $f \geq 0$ on bd $I_{n}$, or $f<0$ on $I_{n}$ and $f \leq 0$ on bd $I_{n}$. Set $\varepsilon_{n}=\min \left\{\inf |f|\left[\mathcal{C}_{f} \cap\right.\right.$ $\left.\left.I_{n}\right]+n^{-1}, T\right\}$ and use Lemma 4.5 to construct functions $g_{1 n}, \ldots, g_{k n} \in \mathbf{D Q} \mathcal{F}$ such that $|f|=g_{1 n} \ldots g_{k n}$ on $I_{n}$ and for each $i$ : $\left\|g_{i n}\right\| \leq T, g_{i n}\left[\mathcal{C}_{g_{i n}} \cap I_{n}\right] \supset$ $\left[\varepsilon_{n} / T^{k-1}, T\right]$ and $g_{i n}=\sqrt[k]{|f|}$ on bd $I_{n}$. Put

$$
t_{i n}= \begin{cases}(-1)^{j} & \text { if } i<k, I_{n} \in \mathcal{J}_{j}, j \in\{1,2\}, \\ (-1)^{(k-1) j} \cdot \operatorname{sgn} f\left[I_{n}\right] & \text { if } i=k, I_{n} \in \mathcal{J}_{j}, j \in\{1,2\}, \\ (-1)^{(k-1) j} \cdot \operatorname{sgn} f\left[I_{n}\right] & \text { if } i=1, I_{n} \in \mathcal{J}_{j}, j \in\{3,4\}, \\ (-1)^{j} & \text { if } i>1, I_{n} \in \mathcal{J}_{j}, j \in\{3,4\}\end{cases}
$$

and notice that $t_{1 n} \ldots t_{k n}=\operatorname{sgn} f\left[I_{n}\right]$.

Fix an $i \in\{1, \ldots, k\}$. Put

$$
g_{i}(x)= \begin{cases}g_{i n}(x) & \text { if } x \in I_{n}, I_{n} \subset[f=0], I_{n} \in \mathcal{J}, \\ t_{i n} g_{i n}(x) & \text { if } x \in \operatorname{cl} I_{n}, I_{n} \subset[f \neq 0], I_{n} \in \mathcal{J}, \\ \sqrt[k]{|f(x)|} \cdot(\operatorname{sgn} f(x))^{1+\operatorname{sgn}(k-i)} & \text { otherwise. }\end{cases}
$$

One can easily verify that $\left\|g_{i}\right\| \leq T$ and $g_{i} \in \mathcal{F}$. To prove that $g_{i} \in \mathbf{D Q}$ fix an $x \in \mathbb{R}$. If $A \cap(x, x+\delta)=\emptyset$ for some $\delta>0$, then clearly $x$ is a Darboux point of $g_{i}$ from the right and $g_{i}$ is quasi-continuous at $x$. Otherwise let $\delta>0$. For $j \in\{1, \ldots, 4\}$ there exists a sequence $\left(I_{j, m}\right) \subset \mathcal{J}_{j}$ such that $\sup I_{j, m} \searrow x$ and $\inf |f|\left[\mathcal{C}_{f} \cap I_{j, m}\right] \rightarrow 0$. Thus

$$
g_{i}\left[\mathcal{C}_{g_{i}} \cap(x, x+\delta)\right] \supset \bigcup_{j=1}^{4} \bigcup_{I_{j, m} \subset(x, x+\delta)} g_{i}\left[\mathcal{C}_{g_{i}} \cap I_{j, m}\right] \supset[-T, T] \backslash\{0\}
$$


so $g_{i}$ is quasi-continuous at $x$. But $g_{i}=0$ on $[f=0] \cap A$ and $\left[g_{i}=0\right] \cap(a, b) \neq \emptyset$ whenever $(a, b) \subset[f=0]$. Using the fact that $x \in \operatorname{cl}([f=0] \cap(x, \infty))$, we obtain $g_{i}[(x, x+\delta)]=[-T, T]$. Hence $x$ is a Darboux point of $g_{i}$ from the right. Similarly we can show that $x$ is a Darboux point of $g_{i}$ from the left.

\section{Products of Strong Świątkowski Functions}

Proposition 5.1. There is a bounded function $f \in \mathbf{D Q B}_{2}$ which cannot be written as the finite product of strong Światkowski functions.

Proof. Let $C$ be the Cantor ternary set. Let $\mathcal{J}_{1}$ and $\mathcal{J}_{2}$ be families of components of $\mathbb{R} \backslash C$ such that $C \cup \bigcup \mathcal{J}_{1} \cup \bigcup \mathcal{J}_{2}=[0,1]$ and $C=\left(\operatorname{cl} \cup \mathcal{J}_{1}\right) \cap\left(\operatorname{cl} \bigcup \mathcal{J}_{2}\right)$. Define $f(x)=(-1)^{j} \varrho(x, C) / \lambda(I)$ if $x \in \operatorname{cl} I$ for some $I \in \mathcal{J}_{j}$ and $j \in\{1,2\}$ and $f(x)=1 / 2$ otherwise. It can be readily verified that $f \in \mathbf{D Q B}_{2}$. Suppose that there exist functions $g_{1}, \ldots, g_{k} \in \dot{\mathbf{S}}_{s}$ such that $f=g_{1} \ldots g_{k}$ on $\mathbb{R}$. First we will verify the following assertion.

For each $i \in\{1, \ldots, k\}$, if $I$ is an open interval with $C \cap I \neq \emptyset$, then there is an open interval $J \subset I$ with $C \cap J \neq \emptyset$ such that either $g_{i} \geq 0$ on $J$ or $g_{i} \leq 0$ on $J$.

Indeed, consider the set $A=\mathcal{C}_{g_{i}} \cap C \cap I$. If $A$ is uncountable, then there is an $x_{0} \in A$ with $g_{i}\left(x_{0}\right) \neq 0$. Consequently, there is an open interval $J \subset I$ such that $x_{0} \in C \cap J$ and either $g_{i}>0$ on $J$ or $g_{i}<0$ on $J$.

Otherwise $A$ is an at most countable $G_{\delta}$ set, so $A$ is nowhere dense in $C$. Hence there is an open interval $J \subset I$ such that $A \cap J=\emptyset \neq C \cap J$. Then $\mathcal{C}_{g_{i}} \cap J \subset \mathbb{R} \backslash C$ and $0 \notin g_{i}\left[\mathcal{C}_{g_{i}} \cap J\right]$. Since $g_{i} \in \dot{\mathbf{S}}_{s}$, so either $g_{i} \geq 0$ on $J$ or $g_{i} \leq 0$ on $J$.

By (9), there is an open interval $J$ such that $C \cap J \neq \emptyset$ and each $g_{i}$ is either nonnegative or nonpositive on $J$. Consequently, the function $f$ is either nonnegative or nonpositive on $J$, too. But $J \cap \bigcup \mathcal{J}_{1} \neq \emptyset \neq J \cap \bigcup \mathcal{J}_{2}$, an impossibility.

Proposition 5.2. If a function $f$ can be written as the product of two strong Światkowski functions, then for all $x<t$, if $f(x) f(t)<0$, then $\underline{\operatorname{LIM}}\left(|f|, x_{0}^{-}\right)=$ $\underline{\operatorname{LIM}}\left(|f|, x_{0}^{+}\right)=0$ for some $x_{0} \in[f=0] \cap(x, t)$.

Proof. Suppose $f=g h$, where $g, h \in \dot{\mathbf{S}}_{s}$. If $x<t$ and $f(x) f(t)<0$, then $g(x) g(t)<0$ or $h(x) h(t)<0$. Without loss we may assume that the first case holds. Since $g \in \dot{\mathbf{S}}_{s}$, there is an $x_{0} \in \mathcal{C}_{g} \cap[g=0] \cap(x, t)$. By Lemma 2.3, there is a sequence $\left(x_{n}\right) \subset \mathcal{C}_{f}$ such that $x_{n} \nearrow x_{0}$ and $\lim _{n \rightarrow \infty} h\left(x_{n}\right)=h\left(x_{0}\right)$, whence $\underline{\operatorname{LIM}}\left(|f|, x_{0}^{-}\right) \leq \lim _{n \rightarrow \infty}\left|f\left(x_{n}\right)\right|=0$. Similarly $\underline{\operatorname{LIM}}\left(|f|, x_{0}^{+}\right)=0$. 
Proposition 5.3. There is a continuous function $g_{1}$ and functions $g_{2}, g_{3} \in \dot{\mathbf{S}}_{s}$ such that $g_{1} g_{2} g_{3}=\operatorname{sgn}$ on $\mathbb{R}$ and $\mathcal{D}_{g_{2}}=\mathcal{D}_{g_{3}}=\{0\}$.

Proof. Define $g_{1}(x)=\arctan x$,

$$
\begin{aligned}
& h(x)= \begin{cases}\arctan x+\operatorname{sgn} x+\sin x^{-1} & \text { if } x \neq 0, \\
0 & \text { otherwise }\end{cases} \\
& g_{2}(x)=\left\{\begin{array}{ll}
h(x) / g_{1}(x) & \text { if } x \neq 0, \\
1 & \text { otherwise, }
\end{array} \quad g_{3}(x)= \begin{cases}\operatorname{sgn} x / h(x) & \text { if } x \neq 0, \\
1 & \text { otherwise. }\end{cases} \right.
\end{aligned}
$$

Proposition 5.4. If a function $f$ can be written as the finite product of bounded strong Światkowski functions, then

$$
\text { for all } x<t \text {, if } f(x) f(t)<0 \text {, then } \mathcal{C}_{f} \cap[f=0] \cap(x, t) \neq \emptyset \text {. }
$$

Proof. Suppose that $f=g_{1} \ldots g_{k}$, where $g_{1}, \ldots, g_{k} \in \dot{\mathbf{S}}_{s}$ are bounded. If $x<t$ and $f(x) f(t)<0$, then $g_{i}(x) g_{i}(t)<0$ for some $i \in\{1, \ldots, k\}$. Since $g_{i} \in \dot{\mathbf{S}}_{s}, \mathcal{C}_{g_{i}} \cap\left[g_{i}=0\right] \cap(x, t) \neq \emptyset$. Using the fact that $g_{1}, \ldots, g_{k}$ are bounded, we obtain $\mathcal{C}_{f} \cap[f=0] \cap(x, t) \neq \emptyset$.

Corollary 5.5. There is a bounded function $h \in \mathbf{D Q}$ with $\mathcal{D}_{h}=\{0\}$ which is the product of a continuous function and a strong Światkowski one and which cannot be written as the finite product of bounded strong Siwigtkowski functions.

Proof. Consider the function $h$ defined in the proof of Proposition 5.3.

Problem. Characterize products of (bounded) strong Świạtkowski functions.

\section{References}

[1] N. Biswas, On some mappings in topological spaces, Bull. Calcutta Math. Soc., 61 (1969), 127-135.

[2] J. Borsík, Products of simply continuous and quasicontinuous functions, Math. Slovaca, 45(4), (1995), 445-452.

[3] A. M. Bruckner and J. G. Ceder, Darboux continuity, Jahresber. Deutsch. Math.-Verein., 67 (1965), 93-117.

[4] J. G. Ceder, On factoring a function into a product of Darboux functions, Rend. Circ. Mat. Palermo (2), 31(1), (1982), 16-22. 
[5] Z. Grande and T. Natkaniec, Lattices generated by $\mathcal{T}$-quasi-continuous functions, Bull. Polish Acad. Sci. Math., 34 (1986), 525-530.

[6] S. Kempisty, Sur les fonctions quasicontinues, Fund. Math., 19 (1932), 184-197.

[7] A. Maliszewski, On the limits of strong Światkowski functions, Zeszyty Nauk. Politech. Łódz. Mat., 27(719), (1995), 87-93.

[8] A. Maliszewski, On the sums of non-negative quasi-continuous functions, Acta Math. Hungar., 69(1-2), (1995), 149-157.

[9] A. Maliszewski, On theorems of Pu \& Pu and Grande, Math. Bohem., 121(1), (1996), 83-87.

[10] T. Natkaniec, Products of quasi-continuous functions, Math. Slovaca, 40(4), (1990), 401-405.

[11] T. Natkaniec, On quasi-continuous functions having Darboux property, Math. Pann., 3(2), (1992), 81-96.

[12] H. P. Thielman, Types of functions, Amer. Math. Monthly, 60(3), (1953), $156-161$. 
